Check for updates

Cite this: RSC Adv., 2017, 7, 39869

\title{
A database assisted protein structure prediction method via a swarm intelligence algorithm
}

\author{
Pengyue Gao, (D) a Sheng Wang, ${ }^{a}$ Jian Lv, ${ }^{\text {b }}$ Yanchao Wang ${ }^{\star a}$ and Yanming Ma*a \\ The complex and rugged potential energy landscape has made protein structure prediction a challenging \\ task in computational biology. Here, we propose an efficient protein structure prediction method \\ combining both template-based and template-free methods. Specifically, the initial protein \\ conformations can be built by a non-redundant protein database and random sampling method with \\ constraints of the secondary structure of the proteins. Three different structure evolution methods \\ including improved particle swarm optimization (PSO) algorithm, random perturbation and fragment \\ substitution are employed to update the protein structures while keeping the secondary structures the \\ same. The present method is benchmarked on several known protein structures with distinct folding \\ patterns, including $\alpha$ proteins, $\beta$ proteins and $\alpha \beta$ proteins. The high success rate and the accuracy of the \\ results demonstrate the reliability of this method.
}

Received 6th July 2017

Accepted 9th August 2017

DOI: 10.1039/c7ra07461a

rsc.li/rsc-advances

structure templates. Comparative modeling identifies

\section{Introduction}

The function of a protein is determined by its threedimensional (3D) structure, and this plays a crucial role in governing certain life processes. ${ }^{1}$ The rapid development of sequencing technologies has resulted in an exponential increase in the number of known protein sequences. However, the experimental determination of protein structure (e.g., by X-ray crystallography and nuclear magnetic resonance spectroscopy) is limited in part by the labor-intensive, lengthy process of protein structure resolution. For example, although more than 70 million known protein sequences have been deposited in the UniProtKB/TrEMBL protein database, ${ }^{2}$ less than $1 \%$ of these protein structures (about 125000 protein structures) have been determined experimentally at the atomic level in the Protein Data Bank (PDB). ${ }^{3}$ This reflects the large gap between identifying the sequence of a protein and determining its structure. Therefore, the development of low-cost and efficient computer-aided methods for predicting protein 3D structures is highly desirable.

Currently, protein structure prediction methods are classified into two categories based on the extent to which they exploit the known experimental structures in the PDB database: template-based method and template-free method. Templatebased method, including comparative modeling ${ }^{4}$ and fold recognition modeling, ${ }^{5,6}$ builds protein models by aligning query sequences on solved protein structures to identify

${ }^{a}$ State Key Laboratory of Superhard Materials, Jilin University, Changchun 130012, China.E-mail:wyc@calypso.cn; mym@jlu.edu.cn

${ }^{b}$ College of Materials Science and Engineering, Jilin University, Changchun 130012, China templates by sequence or sequence profile comparison, since similar sequences adopt similar protein structures. Fold recognition identifies templates by matching the query sequence directly onto known protein structures. Templatebased method has been proven more accurate than templatefree methods. However, it's only successful when templates are available in the PDB library. On the contrary, template-free method, also called $a b$ initio modelling, ${ }^{7-9}$ doesn't rely on any protein structure template and conducts a conformational search under guidance of the energy function to determine the protein structure. According to the thermodynamic hypothesis proposed by Anfinsen, ${ }^{10}$ the native conformation lies at the global free energy minimum. Several ab initio methods $\mathrm{s}^{\mathbf{8}, 9,11-18}$ have been developed and used to determine various protein structures. ${ }^{8,11,14}$ However, with increasing protein sizes, the conformational phase space of sampling sharply increases, which makes the $a b$ initio modeling extremely difficult. In practice, the current trends are pointing to approaches, which can extensively combine both methods. Template-based method always includes exploring template independent conformational space. Similarly, the ab initio modeling builds up models by using fragments of known structures. ${ }^{19-22}$

Here, we develop a general protein structure prediction method enabling the determination of the three-dimensional structure of a protein based on its sequence. The protein sequence is divided into two regions: template-dependent region and template-independent region. The former is constructed by template-based method and the latter is constructed by $a b$ initio method (e.g., random sampling using the secondary structure information). It is also noteworthy that ab initio method is employed for structure evolution. Specifically, all the 
structures will be updated by improved PSO algorithm, fragment substitution method and random perturbation. Due to employment of combination of the template-based and $a b$ initio methods, our method can achieve an adequate trade-off between two opposite terms: exploration and exploitation. The method is applied to several proteins with distinct folding patterns and system sizes. The high success rate and accuracy support the reliability of the method for protein structure prediction.

\section{Method and implementation}

A flow chart for the proposed protein structure prediction method is shown in Fig. 1. It consists of four main steps: (a) preconditioning; (b) construction of initial conformation with the all-atom model of a protein; (c) energy minimization and evaluation of the static energy of the protein 3D structure with the all-atom force field parameters; and (d) generation of evolutionary related structures with the improved PSO algorithm, random perturbation and fragment substitution.

\section{a. Preconditioning}

The aim of preconditioning is selecting the template protein and building a fragment library on basis of the given protein sequence. It's divided into three main steps: secondary structure prediction, threading and building the fragment library.

The secondary structure of proteins is the $3 \mathrm{D}$ form of local segments of proteins. Once the secondary structure is determined, there will be a considerable reduction of the computational cost of structure prediction. Here, one of the widely used secondary structure prediction method: PSIPRED ${ }^{23}$ is adopted in our method.

Threading, as a template-based protein structure prediction method, aims to select template proteins, which share the similar structural motifs with the target protein, from known protein structure databases. The process details are as follows:

First, the query sequence is matched against a nonredundant sequence database (downloaded from http:// zhanglab.ccmb.med.umich.edu/library/) by position-specific iterated BLAST (PSI-BLAST), ${ }^{24}$ to identify sequence similarity.

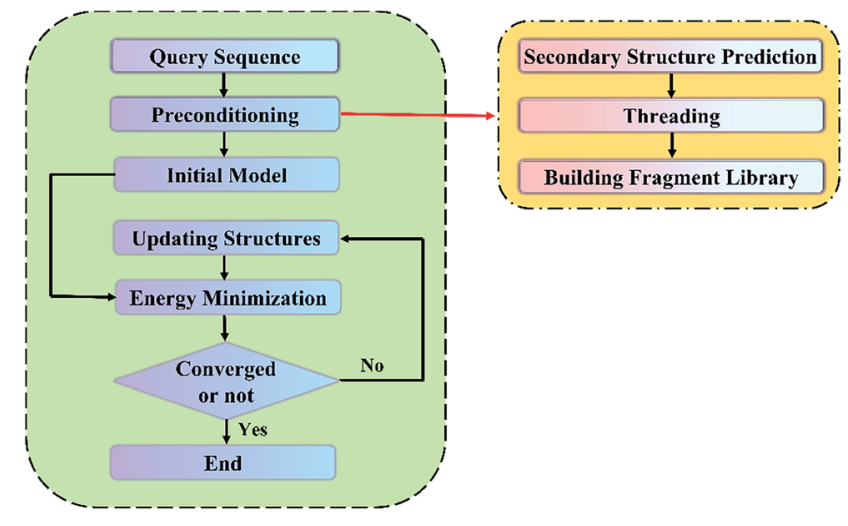

Fig. 1 Flow chart of the protein structure prediction method.
Note that a critical parameter ( $E$-value) is used in the PSI-BLAST search to estimate the probability of sequence similarity and exclude the similar sequences or homologous proteins. In this manuscript, the templates with an $E$-value $<0.05$ are usually excluded to prove the robustness of the method. For a given protein sequence, a large number of target-template alignments can be found by the PSI-BLAST search. In order to select the potential template to construct the initial conformation, all the target-template alignments are evaluated by a score function related to both sequence and structure, borrowed from a profile-profile aligning approach: PPA approach ${ }^{25}$ and ranked according to their scores. Only the structures with the highscore can be selected as the potential templates.

To validate the threading method used in our studies, the protein 1r69_(61 residues) is employed as the benchmark. The sequence of protein 1r69_ as the query sequence is matched against the non-redundant sequence database by PSI-BLAST method and all the target-template alignments are evaluated by PPA approach. A protein alignment (1y7yA) with the highest score of 2.82 is shown in Fig. 2. The C $\alpha$-RMSD between the structures of the native protein 1r69_ (3rd-56th residue) and protein 1y7yA (11th-65th residue) is only $1.4 \AA$ A. Obviously, both of structures share the similar sequence and structural motif, validating the threading method.

It should be emphasized that two key criteria should be suggested for the potential templates to construct the initial protein model: structural similarity and alignment length. To evaluate the structural similarity, the C $\alpha$-RMSD of all targettemplate alignments should be calculated. It is accepted that the template with $\mathrm{C} \alpha$-RMSD of template less than $3.0 \AA$ and alignment length more than half of the target sequence usually leads to high success ratio to predict the native-like protein. Therefore, two conditions should be satisfied for a potential template: the $\mathrm{C} \alpha$-RMSD in the alignment region from template structure to native protein is less than $3.0 \AA$ and the alignment length is larger than half of the target sequence. The score is well established to quantify the alignment length and structural similarity of template, while one of the central challenges is deciding an appropriate and acceptable the cutoff of score to find the potential template protein. To obtain well-defined cutoff of the score, we use the protein 1r69_ as an example to calculate the success rate of finding a potential template with different cutoff. It can be noted that the success rate for the target-template alignments as potential templates dependence on the score is shown in Fig. 3a. The success rate is greater than $80 \%$, when the cutoff is set as 1 . While it drops to $62 \%$ with cutoff as 0.75 . Therefore, the cutoff of score is suggested to be 1 . In order to further test the cutoff setting, we calculate the success rate for other proteins. Just as shown in Fig. 3b, the success rates for all the protein are not lower than $50 \%$. Particularly, seven proteins are greater than $70 \%$. The high success rates reveal that it is reasonable to set 1.0 as the truncation of score for identifying a potential template among all the target-template alignments.

As mentioned before, there usually are a large number of target-template alignments, which share similar structures or structure motifs. To use these information, we try to build 
Fig. 2 Sequence alignment of protein 1r69_ with 1y7yA. The red letter represents the identical residue in the sequence alignment. The sequence identity (dividing the number of identical positions by the whole alignment length) is $25 \%$, which means the remote homology between these two proteins. The homologous proteins with an E-value $<0.05$ during PSI-BLAST search are excluded.

a fragment library on the basis of these templates. The fragment library can be used to update protein models to improve the success rate of structure prediction. The process is as followed: we list all amino acid sequence fragments (seen as segments) with length of 9 residues $^{26}$ in the query sequence sequentially, until the last letter of the query sequence is included. All the templates can be divided into several protein fragments in the same way (no gap is permitted in any fragment). The fragments can be ranked by the alignment score ${ }^{25}$ and 25 fragments with high score for each segment are used to build fragment library. The protein fragment containing 9 residues associates with protein database, while the segment is the amino acid sequence fragment with length of 9 residues in the query sequence. In practice, a segment can be replaced by the protein fragment, which can be randomly selected from 25 fragments in the fragment library.

\section{b. Construction of initial conformation with the all-atom model of a protein}

The all-atom model is used to determine the 3D structure of the protein in the present method. In the model, the 3D structure of a protein consists of the main backbone and the side chains. The main backbone can be constructed by varying the dihedral angles $(\phi$ and $\psi)$ and by fixing bond lengths and angles within idealized geometries. The fixed bond lengths are set at $1.47 \AA$ $(\mathrm{N}-\mathrm{C} \alpha), 1.53 \AA(\mathrm{C} \alpha-\mathrm{C}), 1.32 \AA(\mathrm{C}-\mathrm{N})$, and $1.24 \AA(\mathrm{C}-\mathrm{O})$, and the fixed bond angles are set at $110^{\circ}(\mathrm{N}-\mathrm{C} \alpha-\mathrm{C}), 114^{\circ}(\mathrm{C} \alpha-\mathrm{C}-\mathrm{N}), 123^{\circ}$ $(\mathrm{C}-\mathrm{N}-\mathrm{C} \alpha)$, and $121^{\circ}(\mathrm{C} \alpha-\mathrm{C}-\mathrm{O}){ }^{27}$ Peptide bonds are considered to be in an all-trans conformation, and thus the dihedral angles $(\omega)$ are set at $180^{\circ}$. The side chains are added by using the SCWRL $^{28}$ program for a given backbone structure, relying on a backbone-dependent rotamer library to select the most favorable rotamers.

After the PSI-BLAST search, the query sequence is divided into two regions: template-dependent region and templateindependent region. For the template-dependent region, the dihedral angles of the template are copied to construct the initial conformation. For the template-independent region, the initial conformation is constructed based on results of the secondary structure prediction. The two common secondary structures are $\alpha$-helix and $\beta$-sheet, in which the dihedral angle $\phi / \psi$ values are fixed: $-57^{\circ}$ and $-47^{\circ}$ for $\alpha$-helix and $-139^{\circ}$ and $135^{\circ}$ for $\beta$-sheet, respectively. ${ }^{29}$ Other dihedral angles are randomly generated.

\section{c. Energy minimization and evaluation of the static energy of the protein 3D structure with the all-atom force field parameters}

One common step for protein structure prediction is to sample a large number of possible conformations based on a sequence, followed by energy evaluation of each conformation. Therefore, an efficient, reliable scoring function to evaluate the energies of an ensemble of structures and to rank the structures accordingly is valuable for improving the accuracy of a structure prediction program. To reduce the noise of energy surfaces and generate physically justified structures, energy minimization with an all-atom molecular mechanics force field is one of the most important processes in protein structure prediction for deriving the local minima from a pool of initial structures. Several molecular force fields have been developed for model quality assessment, including AMBER, ${ }^{30-32}$ CHARMM, ${ }^{33-35}$ and GROMOS96, ${ }^{36}$ which are

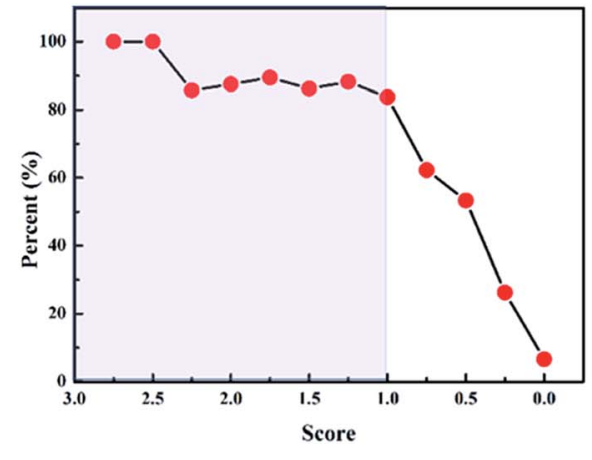

(a)

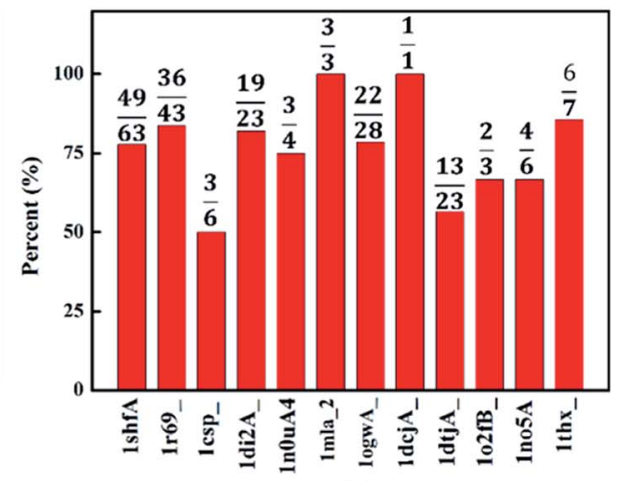

(b)

Fig. 3 (a) The success rate of finding a potential template versus the cutoff of the modelling score for protein 1r69_. (b) The success rate of finding a potential template for several proteins with the modelling score cutoff of value 1 . The fraction above the bar represents the success rate. The denominator represents the number of all the target-template alignments. The numerator represents the numbers of potential templates. 
derived from the laws of physics. In the present study, energy minimization is performed with the GROMACS software package ${ }^{37}$ in which the OPLS force field ${ }^{38}$ is used to describe the interactions between the atoms of a protein by using a GB/SA model ${ }^{39,40}$ for implicit solvation. Additionally, the knowledge-based potential, RWplus, ${ }^{41}$ which has good transferability, is used to calculate the static energy of the protein as the fitness function to guide the evolution processes.

\section{d. Generation of evolutionary related structures with the improved PSO algorithm, random perturbation and fragment substitution}

Efficient sampling of the complex and rugged potential energy surface of protein systems requires multi-dimensional global optimization algorithms. Particle swarm optimization (PSO) algorithm, as an efficient global optimization method, has been successfully applied to predict the structures of clusters, ${ }^{\mathbf{4 2 , 4 3}}$ crystals, ${ }^{44,45}$ two-dimensional layers ${ }^{\mathbf{4 6}}$ and surfaces, ${ }^{47}$ and other systems ${ }^{48}$ which are related to sampling of complex potential energy surface.

In the PSO scheme, a structure (an individual) in the search space is regarded as a particle. A set of individual particles is called a population or a generation. Each particle is initialized at a random position in the search space. During the evolution process, eqn (1) is used to update the positions of particles as

$$
x_{i, j}{ }^{t+1}=x_{i, j}{ }^{t}+\nu_{i, j}{ }^{t+1} \text {. }
$$

The new velocity $\left(\nu_{i, j}{ }^{t+1}\right)$ of the $i$ th individual at the $j$ th dimension is calculated based on its previous location $x_{i, j}{ }^{t}$, previous velocity $\nu_{i, j}{ }^{t}$, current location $\mathrm{pbest}_{i, j}{ }^{t}$ with an achieved best fitness for this individual, and the population global location to date, gbest $^{t}$, with the best fitness value for the entire population according to eqn (2)

$$
\begin{aligned}
\nu_{i, j}{ }^{t+1}= & K \times\left(\nu_{i, j}{ }^{t}+n_{1} \times r_{1} \times\left(\text { pbest }_{i, j}{ }^{t}-x_{i, j}{ }^{t}\right)\right. \\
& \left.+n_{2} \times r_{2} \times\left(\text { gest }^{t}-x_{i, j}{ }^{t}\right)\right)
\end{aligned}
$$

where $K$ is the constriction factor, defined by eqn (3) as

$$
K=\frac{2}{\left|2-\varphi-\sqrt{\varphi^{2}-4 \varphi}\right|}
$$

where $\varphi=n_{1}+n_{2}$, and $r_{1}$ and $r_{2}$ are random numbers ranging from 0 to 1 . Note that the similarity between new structures and pbest/gbest structures depends entirely on the constriction factor. A large constriction factor allows the maximum possible dissimilarity between new structures and $p \mathrm{best} / \mathrm{gbest}$ structures and increases the probability of searching new regions of the solution space; however, it leads to slow convergence to a good solution, whereas a small value increases the convergence, but limits the search regions of solution space. To balance these two effects, $n_{1}$ and $n_{2}$ are usually set at $2.05 .{ }^{49}$ In the traditional PSO method, the variables of all the dimension of each individual are updated for each iteration. For protein structure prediction depending on the template information, the initial conformation usually is similar to the native structure. Traditional PSO method will lead to a bad structure because of changing the conformation on a large scale. So we try to improve the traditional PSO method by only updating one random dimension variable of each individual for each iteration in our protein structure prediction method.

Fragment substitution method uses the structural fragments from the built fragment library to replace the existing part of protein conformations for structure evolution. The position for replacing the protein conformation is randomly selected and the fragment is randomly selected from the 25 fragments. Dihedral angles from the selected fragment replace the dihedral angles in the protein conformation. Fragment substitution can lead to rapid convergence on collapsed structures of plausible topology. Random perturbation method tries to perturb the current dihedral angles, which are randomly selected. In our method, the upper limit of the perturbation is $30^{\circ}$. Random perturbation method can effectively increase the structure diversity.

Note that the secondary structures and bond lengths are frozen, only dihedral angles of coil regions are updated during the structure evolution. Each operated structure is rejected or accepted on the basis of energy criteria. If the energy of the operated structure is lower than the energy of previous structure, the operation is accepted. Otherwise, it is rejected.

To evaluate the performance of different structure evolution strategies, we take protein 1r69_ as an illustrative example. The evolution histories with different structure evolution strategies are shown in Fig. 4a. First, we compare three structure evolution strategies: the traditional PSO algorithm (back line), random perturbation (blue line) and the improved PSO algorithm (red line). During the structure evolution by the traditional PSO algorithm or random perturbation, the system soon stagnates and one or more local minima dominate the search, while the improved PSO algorithm can achieve a fine exploration of potential energy surface and further predict the protein model with lower energy. Therefore, it is more efficient than traditional PSO algorithm or random perturbation. Furthermore, we also consider combination of different structure operations, including the improved PSO algorithm combined with random perturbation (purple line), random perturbation and fragment substitution (green line). The combination of two operations (purple line) can obtain the model with lower energy and it demonstrates that the combination is superior to single one. Especially, the energy decreases fast and the model with lowest-energy is successfully predicted at 225th generation, when the combination of three structure operations is used to perform structure evolution. The model with lowest-energy is proved to be a native-like structure and the $\mathrm{C} \alpha$-RMSD of lowest energy model relative to native protein is only $1.9 \AA$ A. Furthermore, the superposition of lowest-energy model onto the native structure is shown in the Fig. 4b. A subtle difference between the lowest energy model and native structure is insignificant. Obviously, the structure evolution strategy of composite structure operations is the most powerful method to update protein structure. 


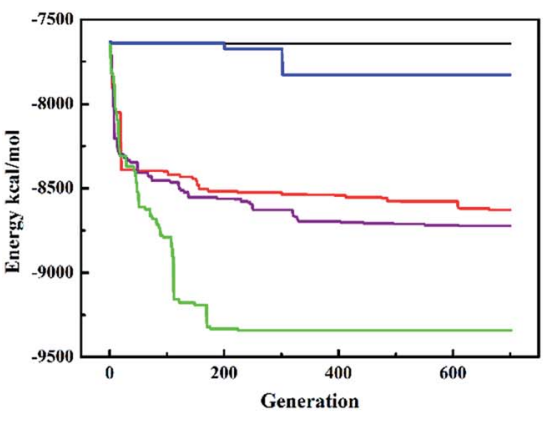

(a)
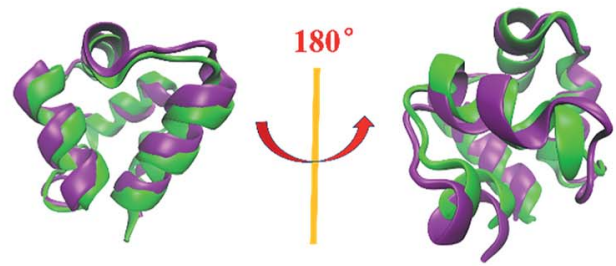

Fig. 4 (a) Evolutionary history of structure search for protein 1r69_. The black line represents the evolution history with traditional PSO algorithm. The red line represents the evolution history with improved PSO algorithm. The blue line represents the history with random perturbation. The purple line represents the evolution history with improved PSO algorithm and random perturbation. The green line represents the evolution history with improved PSO algorithm, random perturbation and fragment substitution. The maximum numbers of generations for the termination condition and swarm size are set as 1000 and 50, respectively. Note that the halting criterion is by default set to 100 further generations if the simulation can't find other better structures or reaching the maximum generations. (b) The superposition of lowest-energy model predicted by the combination of three structure operations onto the native structure of protein 1r69_. The purple represents the native structure and the green represents the predicted lowest-energy model.

\section{Results and discussion}

Generally, the protein structure prediction methods under the guide of homologous proteins will lead to high success rate and accuracy. Therefore, it is less persuasive to prove the robustness of protein prediction method using the template of homologous proteins. So it usually excludes homologous proteins from the structure database when evaluating the performance of protein prediction method. In our studies, we only focus on the results excluding homologous proteins from the database and the criterion for excluding homologous proteins is $E$-value $=0.05$ of the PSI-BLAST search.

The three categories of proteins with distinct folding patterns, including $\alpha$ proteins, $\beta$ proteins and $\alpha \beta$ proteins, are used to evaluate the performance of our approach. The modelling results of several known proteins, with length from 47 residues to 118 residues, are predicted by our approach and shown in Table 1. It is obvious that the C $\alpha$-RMSDs of most targets are less than $10 \AA$, which can be useful for biological application..$^{50}$ Particularly, the $\mathrm{C} \alpha$-RMSD of seven targets of 1shfA, 1r69_, 1di2A, 10gwA, 1o2fB_, 2f3nA and T0716-D1 predicted by our method are less than $2.5 \AA$ (the generally accepted criterion of high-resolution model ${ }^{50}$ ). Thus it can be seen that our method can successfully predict native-like models with different systems sizes and folding patterns, demonstrating the robustness and accuracy of our method.

The accuracy of our method is demonstrated through comparison with the all-atomic ROSETTA ${ }^{53}$ and I-TASSER ${ }^{25}$ methods. Here we take three typical proteins, including $\alpha$ protein (1r69_) and $\alpha \beta$ protein (1di2A_ and 1 thx_) as examples. The protein 1r69_ has a topology of four $\alpha$-helices with 61 amino acid residues. The C $\alpha$-RMSD of the lowest energy model predicted by our method is $1.9 \AA$, which is comparable to the values determined by the ROSETTA $(1.2 \AA)$ and I-TASSER $(1.9 \AA)$ method. The superposition of the lowest model predicted by our method onto the corresponding native structure is shown in
Fig. 5a. A subtle difference observed between the two models demonstrates the accuracy of our method. Furthermore, protein 1di2A_ contains three stands and two helices. The superposition of the lowest-energy model predicted by our method onto the corresponding native structure is shown in the Fig. $5 \mathrm{~b}$. The difference between the two models is negligible. Particularly, the model predicted by our method has the C $\alpha$-RMSD of $1.6 \AA$, which is superior to that obtained by the ROSETTA ( $2.6 \AA)$ and I-TASSER $(2.3 \AA)$. We also test a complex protein 1 thx _ with more than 100 amino acid residues. The C $\alpha$-RMSD of the lowest energy model predicted by our method is $5.9 \AA$ A A native-like structure of target 1 thx difference between the predicted structure and native structure is the relative position of the secondary structures (Fig. 5c). Although the model is not good as that obtained with the I-TASSER method $(2.1 \AA)$, the model has approximately correct topology and is still useful for biological application..$^{50}$ Therefore, the present method is comparable or superior to previously described protein structure search method and can be used to efficiently predict protein structures.

It is well-known that the conformational phase space of sampling sharply increases with protein sizes increasing, which makes the $a b$ initio modeling of proteins with large sizes extremely difficult. Our calculations indicate the accuracy of the models obtained by our method decreases with increasing protein sizes. Just as illustrated in Table 1 , the C $\alpha$-RMSD of proteins with length $>100$ residues (1thx_, 1 jnuA and 1 orgA) predicted by our method are more than $5 \AA$. It is obvious that only the coarse models can be obtained by our method for the proteins, whose lengths are more than 100 residues.

To explore the reasons for the success of our developed method, we perform the structure prediction of two typical proteins of 1 shfa and 1 nouA4. The 1shfA is a typical $\beta$ protein, containing 59 amino acid residues. The $\mathrm{C} \alpha$-RMSD of the lowestenergy model predicted by our method is $2.1 \AA$, which is superior to that obtained by ROSETTA method (10.8 $\mathrm{A})$. In-depth 
Table 1 Summary of our simulations in comparison with ROSETTA and I-TASSER. $N$ is the number of amino acid residues in the protein. $R_{\text {Emin }}$ is the $C \alpha$-RMSD (TM-score ${ }^{51,52}$ ) of the lowest energy structure predicted by our method. The C $\alpha$-RMSD of the lowest energy structure predicted by ROSETTA is list in the fifth column. The C $\alpha$-RMSD (TM-score) of the lowest energy structure predicted by I-TASSER method is list in the sixth column

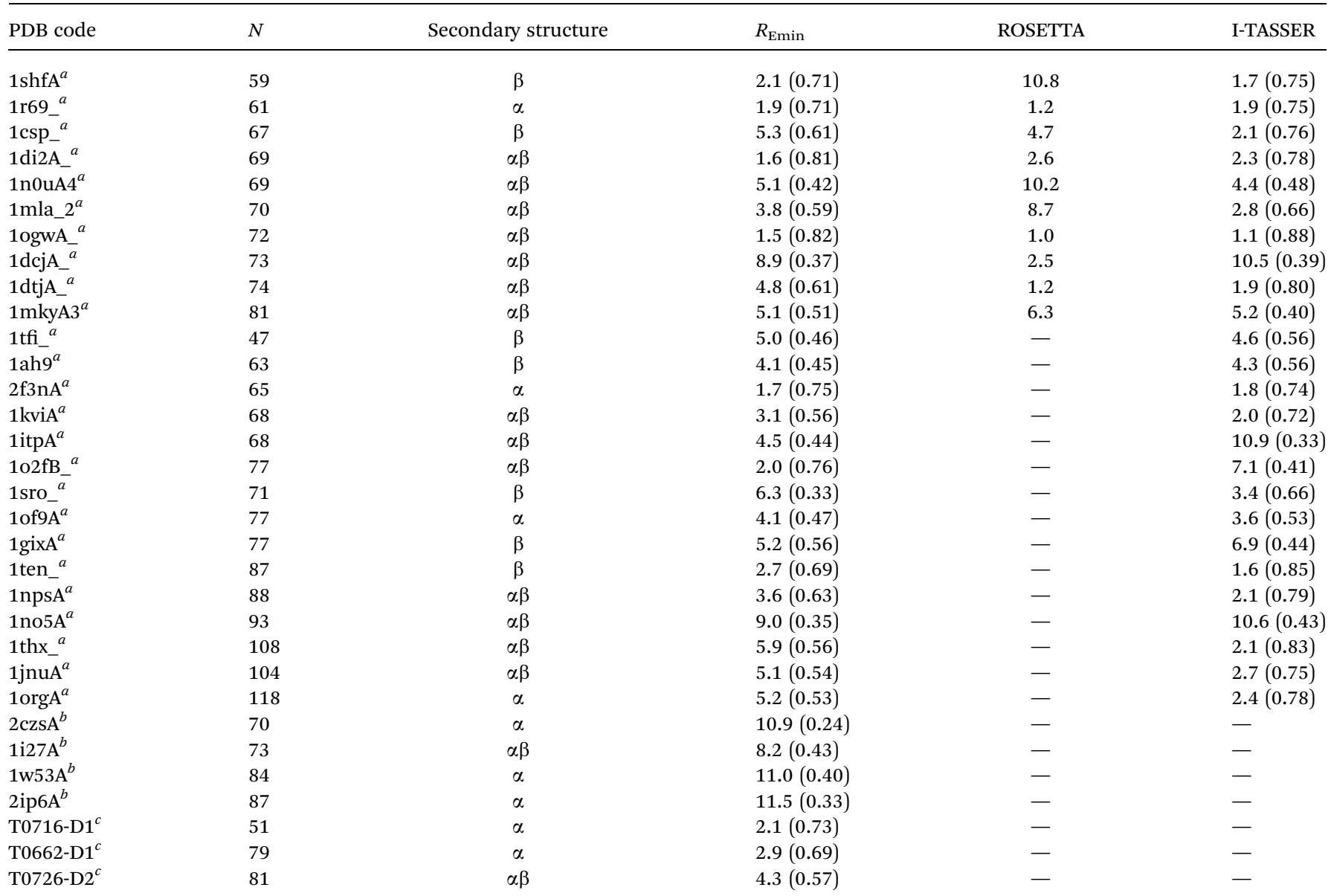

${ }^{a}$ Ref. $25 .{ }^{b}$ Website: http://zhanglab.ccmb.med.umich.edu/decoys/decoy3.html. ${ }^{c}$ Website: http://predictioncenter.org/casp10/index.cgi.

study shows that a high-resolution initial model with the $\mathrm{C} \alpha$-RMSD of $2.5 \AA$ is obtained from the known protein structure databases plays a critical role in successfully predicting the native-like protein. It demonstrates the process of determining the template is very effective for our protein structure prediction. To illustrate the effectiveness of $a b$ initio structure evolution method, we take the protein $1 \mathrm{n} 0 \mathrm{uA} 4$ as an example. Protein

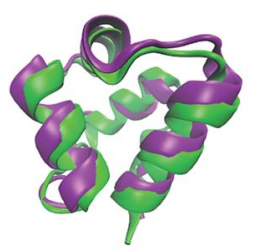

(a)

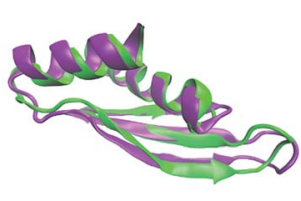

(b)

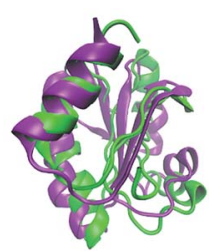

(c)
Fig. 5 The superposition of the native and the lowest-energy struc-

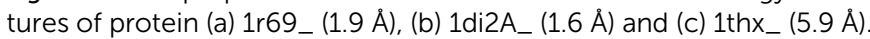
The purple represents the native structure and the green represents the predicted lowest-energy model.
1n0uA4 has the length of 69 amino acid residues with four stands and two helices. The initial model is constructed by selected potential template with the C $\alpha$-RMSD of $6.8 \AA$. The lowest-energy model is predicted at 833th generation with the

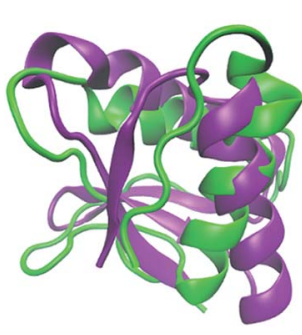

(a)

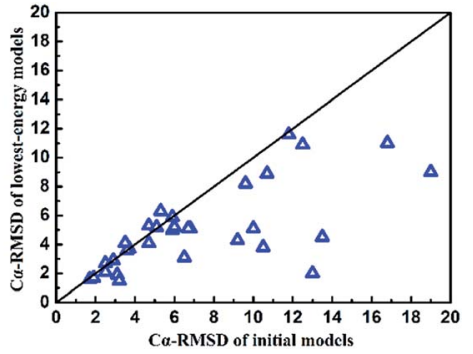

(b)
Fig. 6 (a) The superposition of the native and the lowest-energy structures of protein 1 nOuA4. The purple represents the native structure and the green represents the predicted lowest-energy model. (b) The comparison of C $\alpha$-RMSD between initial models and predicted lowest-energy models of the studied proteins listed in Table 1. 
C $\alpha$-RMSD of $5.1 \AA$, which is superior to the initial model. The superposition of the native and the lowest-energy structures showed in the Fig. 6a indicates that a native-like structure of target 1nouA4 is successfully generated. These results indicate that the structure evolution algorithm adopted in our method can effectively improve the modelling accuracy.

In order to further evaluate the performance of our method, we compare the $\mathrm{C} \alpha$-RMSD of the initial models with the lowestenergy models for all proteins in Table 1. Just as shown in Fig. $6 \mathrm{~b}$, it can be clearly seen that most $\mathrm{C} \alpha$-RMSDs of predicted models are reduced. In other words, the predicted protein conformations become closer to the native proteins than initial ones. These results further demonstrate that our structure evolution algorithm has a powerful ability to improve the modelling accuracy.

\section{Conclusions}

In the manuscript, we propose a protein structure prediction method by combining the templated-based method and ab initio search. Several information including ideal bond lengths and angles, secondary structure constraints and fragment library obtained by known protein database are implemented to substantially reduce the search space. Furthermore, the designed structure operations including an improved PSO algorithm, random perturbation, fragment substitution and their combinations are developed to perform structure evolution. Our method has been implemented in the CALYPSO software package and benchmarked by several known proteins with distinct folding patterns. The native-like structures of these proteins are successfully predicted. The high success rate and accuracy demonstrate the reliability and robustness of this method, which holds promise for narrowing the sequence-structure gap.

\section{Conflicts of interest}

There are no conflicts of interest to declare.

\section{Acknowledgements}

The authors acknowledge the funding support from the National Natural Science Foundation of China under Grant No. 11274136, 11404128, and 11534003, the National Key Research and Development Program of China under Grants No. 2016YFB0201200, the 2012 Changjiang Scholar of the Ministry of Education, the National Key Laboratory of Shock Wave and Detonation Physics and the China Postdoctoral Science Foundations (No. 2015T80294 and No. 2014M551181). Parts of the calculations are performed in the high performance computing center of Jilin University and at Tianhe2-JK in the Beijing Computational Science Research Center.

\section{References}

$1 \mathrm{H}$. Rangwala and G. Karypis, Introduction to protein structure prediction: methods and algorithms, John Wiley \& Sons, Inc, Hoboken, New Jersey, 2010.
2 The UniProt Consortium, Nucleic Acids Res., 2007, 36, D190D195.

3 H. M. Berman, J. Westbrook, Z. Feng, G. Gilliland, T. N. Bhat, H. Weissig, I. N. Shindyalov and P. E. Bourne, Nucleic Acids Res., 2000, 28, 235-242.

4 N. Guex and M. C. Peitsch, Electrophoresis, 1997, 18, 27142723.

5 Y. Xu and D. Xu, Proteins: Struct., Funct., Genet., 2000, 40, 343-354.

6 J. Skolnick, D. Kihara and Y. Zhang, Proteins: Struct., Funct., Genet., 2004, 56, 502-518.

7 A. Liwo, M. Khalili and H. A. Scheraga, Proc. Natl. Acad. Sci., 2005, 202, 2362-2367.

8 J. L. Klepeis, Y. Wei, M. H. Hecht and C. A. Floudas, Proteins: Struct., Funct., Genet., 2005, 58, 560-570.

9 J. Klepeis and C. Floudas, Biophys. J., 2003, 85, 2119-2146.

10 C. B. Anfinsen, Science, 1973, 181, 223-230.

11 R. Das, B. Qian, S. Raman, R. Vernon, J. Thompson, P. Bradley, S. Khare, M. D. Tyka, D. Bhat, D. Chivian, D. E. Kim, W. H. Sheffler, L. Malmström, A. M. Wollacott, C. Wang, I. Andre and D. Baker, in Proteins: Structure, Function and Genetics, 2007, vol. 69, pp. 118-128.

12 Y. Zhang and J. Skolnick, Proc. Natl. Acad. Sci. U. S. A., 2004, 101, 7594-7599.

13 H. Zhou and J. Skolnick, Biophys. J., 2007, 93, 1510-1518.

14 Y. Zhang, Proteins, 2007, 69, 108-117.

15 A. Liwo, J. Lee, D. R. Ripoll, J. Pillardy and H. A. Scheraga, Proc. Natl. Acad. Sci. U. S. A., 1999, 96, 5482-5485.

16 F. L. Custódio, H. J. C. Barbosa and L. E. Dardenne, Appl. Soft Comput. J., 2014, 15, 88-99.

17 A. Verma, A. Schug, K. H. Lee and W. Wenzel, J. Chem. Phys., 2006, 124, 044515.

18 S. Roy, S. Goedecker, M. J. Field and E. Penev, J. Phys. Chem. $B, 2009$, 113, 7315-7321.

19 D. Xu and Y. Zhang, Proteins: Struct., Funct., Bioinf., 2012, 80, 1715-1735.

20 D. Xu and Y. Zhang, Proteins: Struct., Funct., Bioinf., 2013, 81, 229-239.

21 I. Kalev and M. Habeck, Bioinformatics, 2011, 27, 3110-3116. 22 D. T. Jones, Proteins: Struct., Funct., Genet., 2001, 45, 127132.

23 D. T. Jones, J. Mol. Biol., 1999, 292, 195-202.

24 S. F. Altschul, T. L. Madden, A. A. Schäffer, J. Zhang, Z. Zhang, W. Miller and D. J. Lipman, Nucleic Acids Res., 1997, 25, 3389-3402.

25 S. Wu, J. Skolnick and Y. Zhang, BMC Biol., 2007, 5, 17.

26 C. A. Rohl, C. E. M. Strauss, K. M. S. Misura and D. Baker, Methods Enzymol., 2004, 383, 66-93.

27 M. J. Zaki, C. Bystroff and J. Mohammed, Protein structure prediction, Humana Press, New York, USA, 2008.

28 M. J. Bower, F. E. Cohen and R. L. Dunbrack, J. Mol. Biol., 1997, 267, 1268-1282.

29 A. Kukol, Molecular Modeling of Proteins, Humana Press, Hatfield, Hertfordshire, UK, 2015, vol. 1215.

30 S. J. Weiner, P. A. Kollman, D. A. Case, U. C. Singh, C. Ghio, G. Alagona, S. Profeta and P. Weinerl, J. Am. Chem. Soc., 1984, 106, 765-784. 
31 W. D. Cornell, P. Cieplak, C. I. Bayly, I. R. Gould, K. M. Merz, D. M. Ferguson, D. C. Spellmeyer, T. Fox, J. W. Caldwell and P. A. Kollman, J. Am. Chem. Soc., 1995, 117, 5179-5197.

32 Y. Duan and P. A. Kollman, Science, 1998, 282, 740-744.

33 B. R. Brooks, R. E. Bruccoleri, B. D. Olafson, D. J. States, S. Swaminathan and M. Karplus, J. Comput. Chem., 1983, 4, 187-217.

34 E. Neria, S. Fischer and M. Karplus, J. Chem. Phys., 1996, 105, 1902-1921.

35 A. D. MacKerell, D. Bashford, M. Bellott, R. L. Dunbrack, J. D. Evanseck, M. J. Field, S. Fischer, J. Gao, H. Guo, S. Ha, D. Joseph-McCarthy, L. Kuchnir, K. Kuczera, F. T. Lau, C. Mattos, S. Michnick, T. Ngo, D. T. Nguyen, B. Prodhom, W. E. Reiher, B. Roux, M. Schlenkrich, J. C. Smith, R. Stote, J. Straub, M. Watanabe, J. Wiórkiewicz-Kuczera, D. Yin and M. Karplus, J. Phys. Chem. B, 1998, 102, 3586-3616.

36 L. D. Schuler, X. Daura and W. F. Van Gunsteren, J. Comput. Chem., 2001, 22, 1205-1218.

37 D. Van Der Spoel, E. Lindahl, B. Hess, G. Groenhof, A. E. Mark and H. J. C. Berendsen, J. Comput. Chem., 2005, 26, 1701-1718.

38 G. a. Kaminski, R. a. Friesner, J. Tirado-rives and W. L. Jorgensen, J. Phys. Chem. B, 2001, 105, 6474-6487.

39 W. C. Still, A. Tempczyk, R. C. Hawley and T. Hendrickson, J. Am. Chem. Soc., 1990, 112, 6127-6129.
40 D. Qiu, P. S. Shenkin, F. P. Hollinger and W. C. Still, J. Phys. Chem. A, 1997, 101, 3005-3014.

$41 \mathrm{~J}$. Zhang and Y. Zhang, PLoS One, 2010, 5, e15386.

42 C. Steffen, K. Thomas, U. Huniar, A. Hellweg, O. Rubner and A. Schroer, J. Comput. Chem., 2010, 31, 2967-2970.

43 J. Lv, Y. Wang, L. Zhu and Y. Ma, J. Chem. Phys., 2012, 137, 084104.

44 Y. Wang, J. Lv, L. Zhu and Y. Ma, Comput. Phys. Commun., 2012, 183, 2063-2070.

45 Y. Wang, J. Lv, L. Zhu and Y. Ma, Phys. Rev. B: Condens. Matter Mater. Phys., 2010, 82, 1-8.

46 Y. Wang, M. Miao, J. Lv, L. Zhu, K. Yin, H. Liu and Y. Ma, J. Chem. Phys., 2012, 137, 224108.

47 S. Lu, Y. Wang, H. Liu, M.-S. Miao and Y. Ma, Nat. Commun., 2014, 5, 3666.

48 B. Gao, X. Shao, J. Lv, Y. Wang and Y. Ma, J. Phys. Chem. C, 2015, 119, 20111-20118.

49 A. Carlisle and G. Dozier, Proceeding Work. Part. Swarm Optim., 2001, vol. 1, pp. 1-6.

50 Y. Zhang, Curr. Opin. Struct. Biol., 2009, 19, 145-155.

51 Y. Zhang and J. Skolnick, Proteins: Struct., Funct., Genet., 2004, 57, 702-710.

$52 \mathrm{~J} . \mathrm{Xu}$ and Y. Zhang, Bioinformatics, 2010, 26, 889-895.

53 P. Bradley, Science, 2005, 309, 1868-1871. 